\title{
Prognostic value of total tumor volume in patients with nasopharyngeal carcinoma treated with intensity-modulated radiotherapy
}

Shao-Bo Liang ${ }^{1,2+}$, Jian-Jian Teng ${ }^{2 \dagger}$, Xue-Feng Hu${ }^{2}$, Xing-Li Yang ${ }^{1}$, Min Luo ${ }^{1}$, Xiao-Na Fang ${ }^{1}$, Dong-Sheng Liu ${ }^{3}$, Yong Chen ${ }^{1,4^{*}}$ and $\mathrm{Li}^{-W u ~ F u}{ }^{1 *}$

\begin{abstract}
Background: Few studies have evaluated the prognostic value of total tumor volume (TT), which reflects both the primary tumor volume and nodal tumor volume, in NPC. Furthermore, the relationship between TTV and survival remains unknown. The purpose of this study was to evaluate the prognostic value of TTV in patients with NPC treated with intensity-modulated radiation therapy (IMRT).
\end{abstract}

Methods: TTV was retrospectively assessed in 455 patients with newly diagnosed, non-metastatic NPC. All patients were treated using IMRT; 91.1\% (288/316) of patients with stage III-IVb also received cisplatin-based chemotherapy. Receiver operating characteristic (ROC) curves were used to identify the optimal TTV cut-off point and examine the prognostic value of combined TTV with current clinical stage.

Results: Mean TTV was $11.1 \mathrm{~cm}^{3}$ (range, $0.3-27.9 \mathrm{~cm}^{3}$ ) in stage I, $22.5 \mathrm{~cm}^{3}\left(1.3-92.4 \mathrm{~cm}^{3}\right)$ in stage $11,40.6 \mathrm{~cm}^{3}$ in stage III $\left(3.2-129.2 \mathrm{~cm}^{3}\right)$, and $77.5 \mathrm{~cm}^{3}$ in stage IVa-b $\left(7.1-284.1 \mathrm{~cm}^{3}\right)$. For all patients, the 4-year estimated FFS, OS, DMFS, and LRRFS rates for patients with a TTV $\leq 28$ vs. $>28 \mathrm{~cm}^{3}$ were 93 vs. $71.4 \%(P<0.001), 95.1$ vs. $75.4 \%(P<0.001), 94.5$ vs. $79.4 \%(P<0.001)$, and 96.2 vs. 88\% ( $P=0.001)$. TTV was an independent prognostic factor for FFS, OS, DMFS and LRRFS in all patients. In stage III-IVb, 4-year estimated FFS, OS, DMFS, and LRRFS for a TTV $\leq 28 \mathrm{vs.}>28 \mathrm{~cm}^{3}$ were 88.9 vs. $70.5 \%$ $(P=0.001), 96.2$ vs. $72.7 \%(P<0.001), 91.2$ vs. $78.3 \%(P=0.008)$, and 93.8 vs. $87.6 \%(P=0.063)$. TTV was an independent prognostic factor for FFS, OS and DMFS in stage III-IVb. Receiver operating characteristic (ROC) curve analysis curves revealed adding TV to clinical stage had superior prognostic value for treatment failure compared to clinical stage alone $(P=0.016)$.

Conclusions: TTV is an important prognosticator for treatment outcome and significantly improves the prognostic value of the current staging system for patients with NPC treated with IMRT.

Keywords: Nasopharyngeal carcinoma, Intensity-modulated radiotherapy, Tumor volume, Treatment failure, Staging system

\footnotetext{
* Correspondence: chenyong@sysucc.org.cn; Fulw@mail.sysu.edu.cn

${ }^{\dagger}$ Equal contributors

${ }^{1}$ State Key Laboratory of Oncology in South China, Sun Yat-sen University

Cancer Center, Collaborative Innovation Center for Cancer Medicine,

Guangzhou 510060, China

Full list of author information is available at the end of the article
} 


\section{Background}

Based on GLOBOCAN estimates, there were an estimated 86,700 new cases of nasopharyngeal carcinoma (NPC) and 50,800 associated deaths worldwide in 2012 [1]. The geographic distribution of NPC is extremely unbalanced, with a very low incidence in most regions of the world and high incidence in China and other countries in Southeastern Asia [1,2]. Radical radiotherapy (RT) is the first treatment choice for non-metastatic NPC and the addition of concomitant chemotherapy to RT provides a significant survival benefit in locoregionally advanced NPC [3].

The overall survival (OS) of patients with NPC has significantly improved in recent years due to widespread application of magnetic resonance imaging (MRI), improvements in RT techniques and the combination of RT with concomitant chemotherapy [4-6]. The 5-year estimated OS rate is currently about $80 \%$, while treatment failure remains the predominant cause of death; 5-year local control ranges from 86 to 95\%, 5-year nodal control from 92 to $97 \%$ and 5-year distant control from 82 to $85 \%$ [7-12].

Accurate prognostication is critical when deciding treatment strategies. Tumor volume is a significant independent prognostic factor in most cancers, including oral carcinoma, B-cell lymphoma and rhabdomyosarcoma [13-15]. Several studies have confirmed the primary tumor volume (PTV) has high prognostic value for survival in NPC $[16,17]$. However, few studies have evaluated the prognostic value of the total tumor volume (TTV), which incorporates both the PTV and nodal tumor volume (NTV), in NPC and the relationship between the TTV and survival remains unknown.

Therefore, we initiated a retrospective, large cohort study to evaluate the prognostic value of TTV in patients with NPC treated with intensity-modulated radiation therapy (IMRT), and assessed whether the prognostic validity of the current staging system for NPC could be improved by incorporating assessment of the TTV. We hope this information may help to further clarify the biological characteristics of NPC and guide the design of individual treatment strategies.

\section{Methods}

\section{Patient characteristics}

The Institutional Review Board of First People's Hospital of Foshan Affiliated to Sun Yat-sen University approved this retrospective study; as this was an analysis of routine clinical data, an exemption from requiring written informed consent was granted. The authenticity of this article has been validated by uploading the key raw data onto the Research Data Deposit public platform (www.researchdata.org.cn), with the approval RDD number as RDDA2017000217. A total of 455 patients with newly diagnosed, non-metastatic NPC treated by IMRT at First People's Hospital of Foshan Affiliated to Sun Yat-sen University from April 2010 to March 2014 were enrolled in this study [18]. The patients included 347 (76.3\%) males and 108 (23.7\%) females. The median age was 45 years (17-80 years). All cases had the non-keratinizing pathological type.

Pretreatment examinations included a medical history, physical examination, hematology and biochemistry profiles, electrocardiogram, chest X-ray, abdominal ultrasound, nasal endoscopy and biopsy, pathological examination of the primary tumor, bone scan, and MRI of the nasopharynx and neck. All patients were restaged using the 7th edition of the American Joint Commission on Cancer staging system (AJCC) [19]. The stage/category distribution for the entire cohort was as follows: 127/455 (27.9\%) in T1, 59 (13.0\%) in T2, 157 (34.5\%) in T3 and 112 (24.6\%) in T4; 58 (12.7\%) in N0, $255(56.0 \%)$ in N1, $119(26.2 \%)$ in N2 and $23(5.1 \%)$ in N3; $29(6.4 \%)$ in stage I, 110 (24.2\%) in stage II, $184(40.4 \%)$ in stage III and $132(29.0 \%)$ in stage IVa-b.

\section{Tumor volume measurement}

The patients were immobilized in a supine position using a thermoplastic mask extending from the head to shoulders. CT simulation (Brilliance Big Bore, Phillips, Amsterdam, Netherlands) was performed at a slice thickness of $3 \mathrm{~mm}$ from the head to $2 \mathrm{~cm}$ below the sternoclavicular joint. The control CT and contrastenhanced CT images were transferred to the inverse IMRT planning system (Version 8.6, Eclipse, Varian, CA, USA). Tumor volumes were delineated by a radiation oncologist, and verified by another radiation oncologist who specializes in NPC treatment.

The PTV and NTV were both delineated on the planning system according to the pretreatment MRI. The PTV included the primary tumor and retropharyngeal lymph node (RLN) involvement as these anatomical sites are so close that it remains difficult to distinguish between them (Fig. 1a-b) [16, 20, 21]. The NTV included metastatic cervical lymph nodes (CLN) and nodal extracapsular spread (Fig. 1c-d). The metastatic lymph nodes were diagnosed based on the criteria recommended by Van et al. and Mao et al. [22, 23]. The diagnostic criteria for nodal extracapsular spread included blurred margins or irregular capsular enhancement of lymph nodes, or tumor invasion into adjacent fat and muscle (Fig. 1c). The PTV and NTV were automatically calculated using a shape-based interpretation algorithm, which is obtained by tri-linear interpolation of a stack of two-dimensional distance transforms of transaxial shapes. The TTV was obtained by summing the PTV and NTV. 

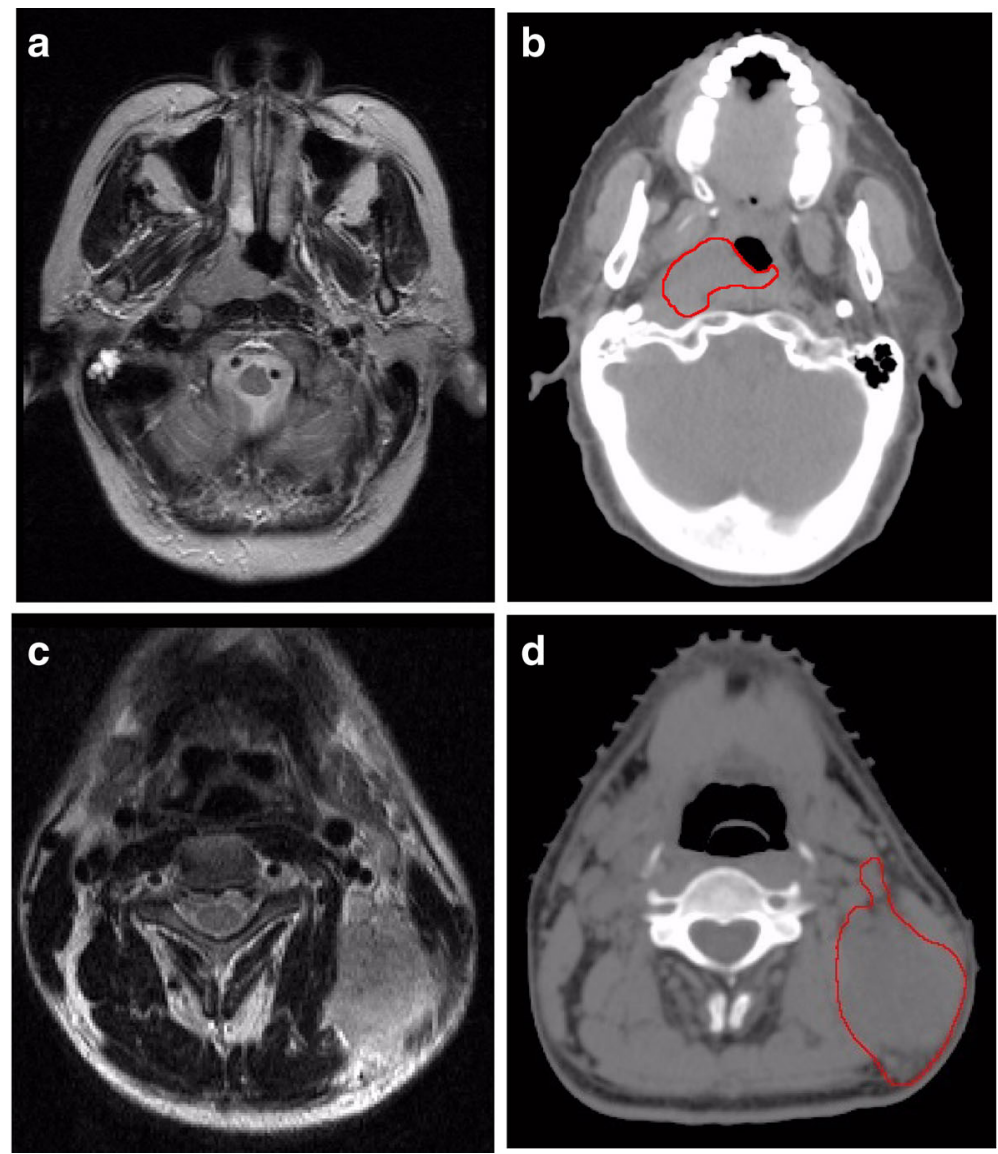

Fig. 1 The delineation of PTV and NTV. a Axial T2-weighted image illustrating that the primary tumor and retropharyngeal lymph node involvement are located close together, making it difficult to distinguish between them. $\mathbf{b}$ Control $\subset T$ image showing the PTV, including the primary tumor and retropharyngeal lymph node involvement, which was delineated according to the pretreatment MRI shown in Fig. 1a. c Axial T2-weighted image of neck lymph nodes with extracapsular spread, which was diagnosed on the basis of an irregular border and invasion into the adjacent fat and muscle. d Control CT image showing the NTV including metastatic cervical lymph nodes and nodal extracapsular spread, which was delineated according to the pretreatment MRI shown in Fig. 1C

\section{Treatment}

All patients were treated using IMRT. Target volumes were delineated according to the RTOG IMRT protocols [18]. The planning target volume of the clinical target volume (CTV)70 received 70 Gy in 33 fractions at 2.12 Gy per fraction. Small-volume lymph nodes received 63 Gy in 33 fractions at 1.9 Gy per fraction. The planning target volume of the CTV59.4 received 59.4 Gy in 33 fractions at 1.8 Gy per fraction. The planning target volume of the CTV50.4 received 50.4 Gy in 28 fractions at 1.8 Gy per fraction. RT was delivered over one fraction daily, 5 days per week.

Based on the treatment guidelines for NPC at our hospital, concurrent chemotherapy was recommended to patients with stage $\mathrm{T} 1-2 \mathrm{~N} 1 \mathrm{M} 0$ and concurrent chemotherapy +/- induction chemotherapy or adjuvant chemotherapy to patients with stage III-IVb NPC. In total, $82(82 / 107,76.6 \%)$ patients with clinical stage
T1-2N1M0 and $288(288 / 316,91.1 \%)$ patients with stage III-IVb received chemotherapy. Induction chemotherapy or adjuvant chemotherapy was consisted of cisplatin $\left(80 \mathrm{mg} / \mathrm{m}^{2}\right)$ and fluorouracil $\left(1000 \mathrm{mg} / \mathrm{m}^{2}\right.$ daily for 4 days); docetaxel $\left(75 \mathrm{mg} / \mathrm{m}^{2}\right)$ and cisplatin $\left(75 \mathrm{mg} / \mathrm{m}^{2}\right)$; or a triplet of docetaxel $\left(60 \mathrm{mg} / \mathrm{m}^{2}\right)$, cisplatin $\left(60 \mathrm{mg} / \mathrm{m}^{2}\right)$ and fluorouracil $\left(800 \mathrm{mg} / \mathrm{m}^{2}\right.$ daily for 4 days) every 3 weeks for 2-3 cycles. Concurrent chemotherapy was consisted of cisplatin given every 3 weeks $\left(100 \mathrm{mg} / \mathrm{m}^{2}\right)$ or weekly $\left(40 \mathrm{mg} / \mathrm{m}^{2}\right)$ during RT. In the event of documented relapse, salvage treatments including RT, surgery or chemotherapy were provided when appropriate.

\section{Follow up and statistical analysis}

After RT, all patients were assessed every 3 months during the first 2 years, and every 6 months thereafter until death. The median follow-up for the entire cohort was 
53 months (range, 2 to 83 months). Overall, 439 patients $(439 / 455,96.5 \%)$ received regular follow-up until death or latest scheduled assessment. Failure free survival (FFS) was calculated from assignment to the first failure at any site, OS to death from any cause, distant metastasis-free survival (DMFS) to first remote failure, and loco-regional relapse free survival (LRRFS) to first locoregional failure.
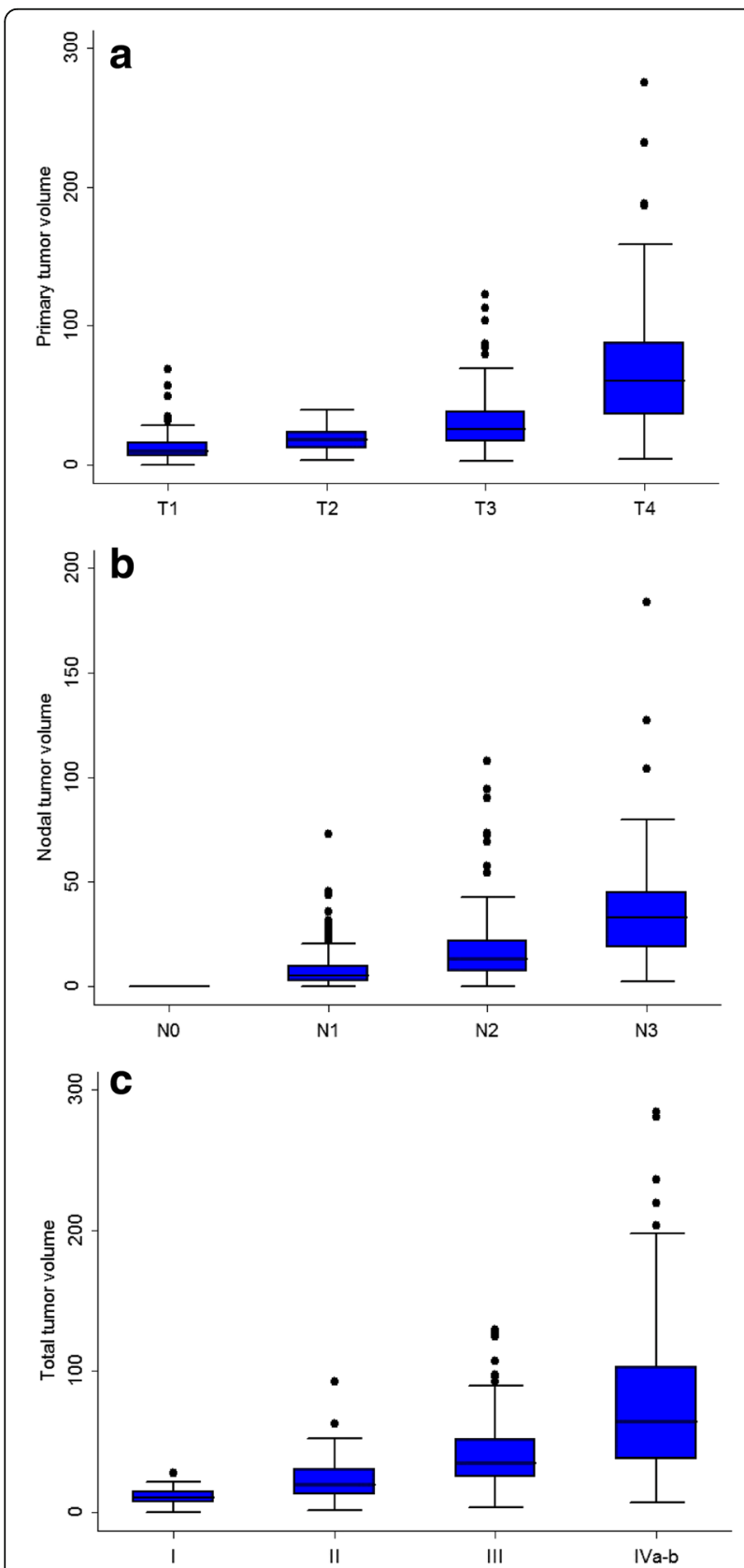

Fig. 2 Distribution of tumor volume by stage for all 455 patients. a Distribution of primary tumor volume by T category. $\mathbf{b}$ Distribution of nodal tumor volume by $\mathrm{N}$ category. $\mathbf{c}$ Distribution of total tumor volume by clinical stage
Stata Statistical Package (STATA 11; StataCorp LP, College Station, TX, USA) was used for all analysis. The Kruskal-Wallis test was used to examine the differences in TTV between stages. Actuarial rates were calculated using the Kaplan-Meier method and compared using the log-rank test. Multivariate analyses with the Cox proportional hazards model were used to test for significant independent prognostic factors using a backward elimination strategy. All patients were randomly allocated to a training set $(n=152)$ or test set $(n=303)$. Receiver operating characteristic (ROC) curve analysis was used to evaluate different cut-off points for TTV in the training set. Then, the test set and all patients were stratified according to the optimal cut-off point. The area under the ROC curve was used to assess the

Table 1 Clinical characteristics of 455 patients with TT $\leq 28$ and TTV $>28 \mathrm{~cm}^{3}$

\begin{tabular}{llll}
\hline Characteristics & $\begin{array}{l}\text { TTV } \leq 28 \mathrm{~cm}^{3} \\
(N=188)\end{array}$ & $\begin{array}{l}\text { TTV }>28 \mathrm{~cm}^{3} \\
(N=267)\end{array}$ & $P$ Value $^{+}$ \\
\hline Sex $(\%)$ & & & $<0.001$ \\
Male & 127 & 220 & \\
Female & 61 & 47 &
\end{tabular}

Age (years) 0.908

$\leq 45$ years

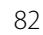

115

$>45$ years

106

152

T-category ${ }^{\mathrm{a}}(\%)$

$\mathrm{T} 1$

$\mathrm{T} 2$

$\mathrm{T3}$

T4

$\mathrm{N}$-category ${ }^{\mathrm{a}}(\%)$

$<0.001$

NO $43 \quad 15$

$\begin{array}{lll}\text { N1 } & 116\end{array}$

N2 $26 \quad 93$

N3

320

Stage-group ${ }^{\mathrm{a}}(\%)$

$<0.001$

$$
\text { । }
$$

II

III

IVa-b

93

105

100

15

20

$29 \quad 0$

$78 \quad 32$

$66 \quad 118$

$15 \quad 117$

Chemotherapy

Yes $\quad 139$

245

$<0.001$

No

49

22

Additional boost

Yes

28

22

0.025

No

160

245

$\pi T V$ total tumor volume; $+P$ values were calculated by the Chi-square test;

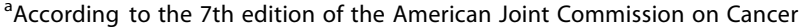
staging system 
prognostic validity of the TTV. The criterion for statistical significance was set at $\alpha=0.05 ; P$-values were based on two-sided tests.

\section{Results}

\section{Distribution of tumor volume by category/stage}

The distribution of PTV stratified by T category is presented in Fig. 2a. The mean PTV was $12.7 \mathrm{~cm}^{3}$ (range, $\left.0.3-69.2 \mathrm{~cm}^{3}\right)$ in T1, $18.9 \mathrm{~cm}^{3}\left(3.2-40 \mathrm{~cm}^{3}\right)$ in T2, $30.7 \mathrm{~cm}^{3}$ in T3 $\left(2.4-122.5 \mathrm{~cm}^{3}\right)$, and $68.7 \mathrm{~cm}^{3}$ in T4 $\left(4.1-275.3 \mathrm{~cm}^{3}\right)$. The distribution of NTV by $\mathrm{N}$ category is presented in Fig. 2b. The mean NTV was $8.0 \mathrm{~cm}^{3}\left(0-72.9 \mathrm{~cm}^{3}\right)$ in $\mathrm{N} 1,18.4 \mathrm{~cm}^{3}$ in $\mathrm{N} 2(0.3-$ $\left.107.5 \mathrm{~cm}^{3}\right)$, and $44.7 \mathrm{~cm}^{3}$ in N3 $\left(2.4-184.0 \mathrm{~cm}^{3}\right)$. The distribution of TTV by clinical stage is presented in
Fig. 2c. The mean TTV was $11.1 \mathrm{~cm}^{3}$ (range, 0.3$\left.27.9 \mathrm{~cm}^{3}\right)$ in stage I, $22.5 \mathrm{~cm}^{3}\left(1.3-92.4 \mathrm{~cm}^{3}\right)$ in stage II, $40.6 \mathrm{~cm}^{3}$ in stage III $\left(3.2-129.2 \mathrm{~cm}^{3}\right)$, and $77.5 \mathrm{~cm}^{3}$ in stage IVa-b $\left(7.1-284.1 \mathrm{~cm}^{3}\right)$.

\section{Identification and verification of TTV cut-off point}

With respect to FFS, the optimal cut-off point for the TTV was $28 \mathrm{~cm}^{3}$ in the training set (sensitivity $95.7 \%$, specificity 50.4\%; area under the ROC curve $[$ AUC] $=0.73$, $P=0.001)$. Therefore, we selected $28 \mathrm{~cm}^{3}$ as a uniform cut-off point ( $\leq 28$ vs. $>28 \mathrm{~cm}^{3}$ ) in order to classify the test set and all patients into high and low TTV groups for survival analysis.

In the test set $(n=303)$, the 4-year estimated FFS, OS, DMFS, and LRRFS rates for patients with a TTV $\leq 28$

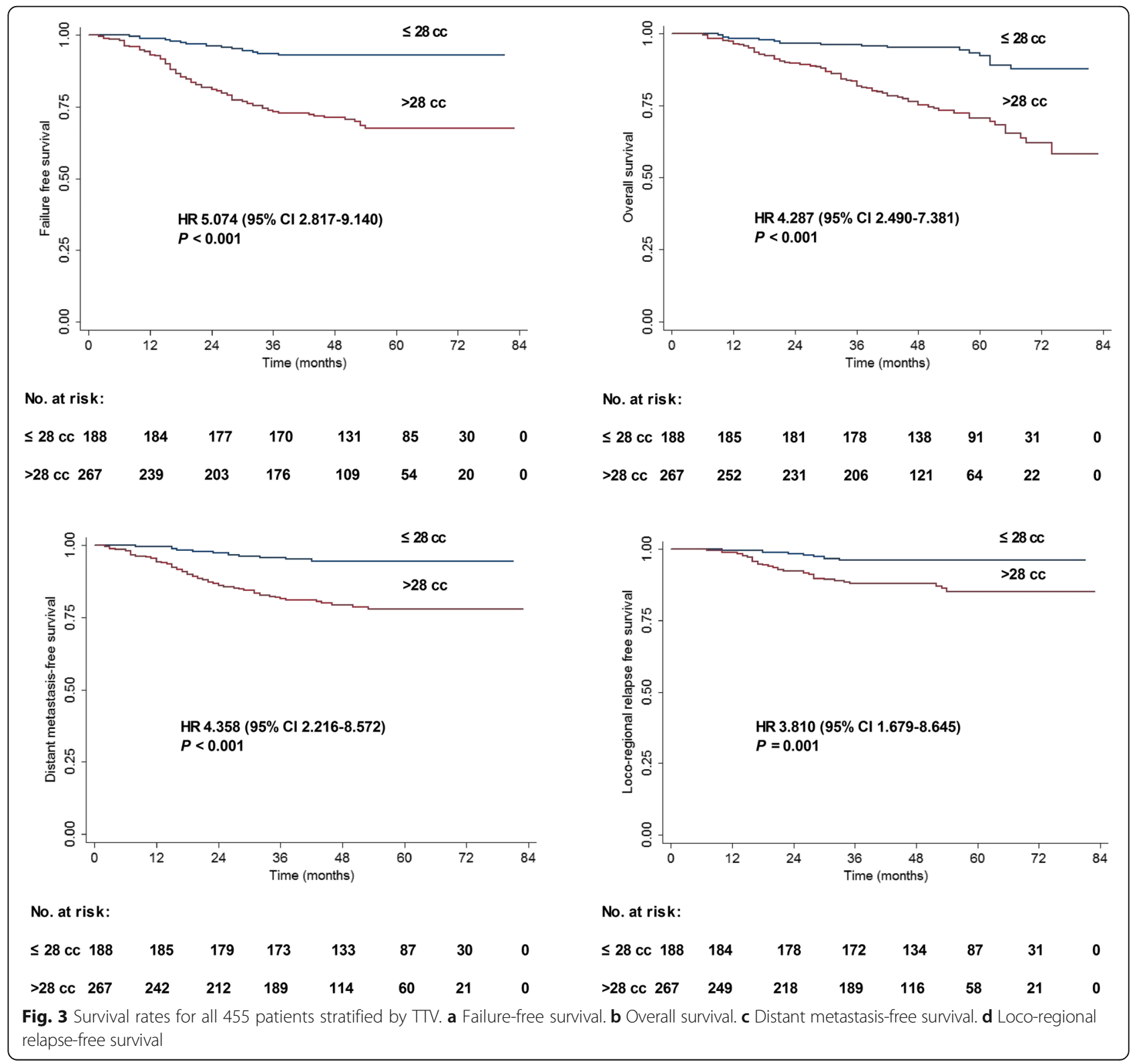


vs. $>28 \mathrm{~cm}^{3}$ were 90.9 vs. $69.8 \%(P<0.001)$, 95.1 vs. 75.1\% $(P<0.001)$, 93.4 vs. $77.8 \%(P<0.001)$, and 95.8 vs. $88.1 \%(P=0.005)$, respectively.

\section{Prognostic significance of TTV in all patients}

The clinical characteristics of the 455 patients with NPC stratified by TTV $\leq 28 \mathrm{~cm}^{3}$ and $>28 \mathrm{~cm}^{3}$ are shown in Table 1 . In all patients $(n=455)$, the 4 -year estimated FFS, OS, DMFS, and LRRFS rates for patients with a TTV $\leq 28$ vs. $>28 \mathrm{~cm}^{3}$ were 93 vs. $71.4 \%(P<0.001)$, 95.1 vs. $75.4 \%(P<0.001), 94.5$ vs. $79.4 \%(P<0.001)$, and 96.2 vs. $88 \%(P=0.001)$, respectively (Fig. 3$)$.

The following parameters were included in the Cox proportional hazards model: age ( $\leq 45$ vs. $>45$ years), sex (male vs. female), T category (T1-2 vs. T3-4), $\mathrm{N}$ category (N0-1 vs. N2-3), chemotherapy (yes vs. no), additional boost (yes vs. no) and TTV ( $\leq 28$ vs. $>28 \mathrm{~cm}^{3}$ ). TTV was an independent prognostic factor for FFS, OS, DMFS and LRRFS in all patients (all $P<0.05$; Table 2).

\section{Prognostic significance of TTV in stage III-IVb NPC}

The 316 patients with stage III-IVb were divided into two subgroups: patients with a TTV $\leq 28 \mathrm{~cm}^{3}(n=81)$ and patients with a TTV $>28 \mathrm{~cm}^{3}(n=235)$. The 4-year estimated FFS, OS, DMFS, and LRRFS rates of the patients with a TTV $\leq 28 \mathrm{~cm}^{3}$ and TTV $>28 \mathrm{~cm}^{3}$ were 88.9 vs. $70.5 \%(P=0.001), 96.2$ vs. $72.7 \%(P<0.001), 91.2$ vs. 78.3\% ( $P=0.008)$, and 93.8 vs. $87.6 \%(P=0.063$; Fig. 4$)$.

The following parameters were included in the Cox proportional hazards model: age ( $\leq 45$ vs. $>45$ years), sex (male vs. female), T category (T1-2 vs. T3-4), N category (N0-1 vs. N2-3), chemotherapy (yes vs. no), additional boost (yes vs. no) and TTV ( $\leq 28$ vs. $>28 \mathrm{~cm}^{3}$ ). TTV was an independent prognostic factor for FFS, OS and DMFS in stage III-IVb NPC (all $P<0.05$; Table 3 ).

Table 2 Multivariate analyses of prognostic factors in all 455 patients

\begin{tabular}{lllll}
\hline Endpoint & Variable & HR & $95 \% \mathrm{Cl}$ & $P$-value \\
\hline FFS & TTV & 4.523 & $2.482-8.241$ & $<0.001$ \\
& N stage $^{\mathrm{a}}$ & 1.567 & $1.027-2.391$ & 0.037 \\
OS & Sex & 1.661 & $0.915-3.013$ & 0.095 \\
& Chemotherapy & 1.882 & $0.986-3.592$ & 0.055 \\
& T stage $^{\mathrm{a}}$ & 1.763 & $1.054-2.951$ & 0.031 \\
& N stage $^{a}$ & 1.920 & $1.254-2.939$ & 0.003 \\
& TTV & 3.231 & $1.776-5.878$ & $<0.001$ \\
DMFS & N stage & 1.764 & $1.064-2.925$ & 0.028 \\
& TTV & 3.749 & $1.877-7.489$ & $<0.001$ \\
LRRFS & TTV & 3.810 & $1.679-8.645$ & 0.001
\end{tabular}

according to the 7th edition of the American Joint Commission on Cancer staging system; $H R$ hazard ratio, $C l$ confidence interval, FFS failure free survival, OS overall survival, DMFS distant metastasis-free survival, LRRFS loco-regional relapse free survival, TTV total tumor volume
Prognostic validity of clinical stage combined with TTV vs. clinical stage alone for treatment failure

ROC curves were used to compare the prognostic validity of clinical stage combined with TTV vs. clinical stage alone for treatment failure. The AUC for clinical stage combined with TTV was 0.706 compared to 0.667 for clinical stage alone $(P=0.016$; Fig. 5$)$. Therefore, the addition of TTV to clinical stage was superior to clinical stage alone for predicting treatment failure.

\section{Discussion}

Tumor size is an important prognostic factor in cancer treatment and has been adopted in the staging systems for most carcinomas [19]. This NPC study demonstrated that patients with a TTV $>28 \mathrm{~cm}^{3}$ had significantly poorer survival outcomes compared to those with a TTV $\leq 28 \mathrm{~cm}^{3}$. Moreover, TTV was an independent prognostic factor in patients with NPC, and the addition of TTV to clinical stage was superior to clinical stage alone for predicting treatment failure.

\section{Distribution and optimal cut-off point for TTV}

High TTV values were more frequent in patients with advanced clinical stage. However, the distribution of the TTV values varied widely within the same clinical stage, and overlapped between different clinical stages. Moreover, TTV, PTV and NTV exhibited large variations between different $\mathrm{T}$ and $\mathrm{N}$ categories [20, 24]. Our previous studies demonstrated that the distribution of the maximum primary tumor diameter (MPTD), another index of tumor size, exhibits a similar trend [25, 26]. Therefore, the current staging system for NPC has the disadvantage of assessing tumor size poorly.

Previous studies have divided patients into 2-4 groups on the basis of tumor volume using different methods $[16,27,28]$. Standard cutoff points should be adopted to achieve optimal sensitivity and specificity. For cancer patients at high risk of treatment failure, it is reasonable to maximize sensitivity over specificity. Therefore, we defined the ideal cut-off point based on a sensitivity estimate of over $80 \%$. A cut-off point of $28 \mathrm{~cm}^{3}$ for the TTV was selected to assess treatment failure, and this cut-off value was validated in the test set.

\section{Prognostic value of the TTV in all patients with NPC}

This study confirmed a large TTV was not only associated with poor FFS, DMFS and LRRFS, but also with poor OS in all patients with NPC. Chua et al. reported the 5-year FFS rates for patients with NPC treated by two-dimensional RT $(2 \mathrm{D}-\mathrm{RT})$ with a TTV $\leq 20 \mathrm{~cm}^{3}$, > $20-40 \mathrm{~cm}^{3},>40-60 \mathrm{~cm}^{3}$ and $>60 \mathrm{~cm}^{3}$ were $89,84,76$ and 55\%, respectively $(P<0.001)$; and the corresponding 5 -year DMFS rates were $84,82,73$ and $61 \%$, respectively $(P<0.001)$ [20]. Thus, it can be concluded that survival 

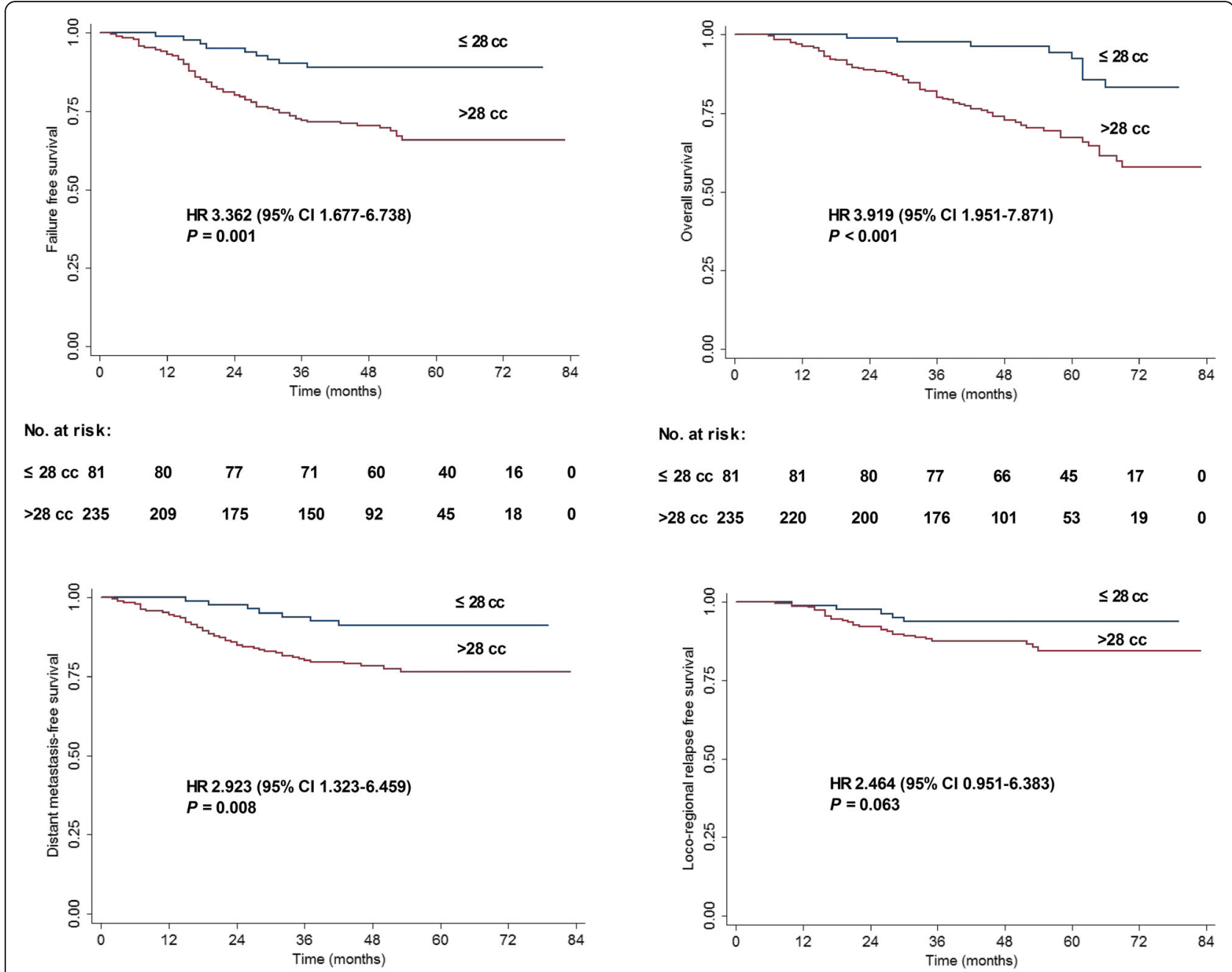

No. at risk:

$\begin{array}{rrrrrrrr}\leq 28 \text { cc } 81 & 81 & 79 & 73 & 61 & 41 & 17 & 0 \\ >28 \text { cc } 235 & 212 & 182 & 160 & 95 & 49 & 18 & 0\end{array}$

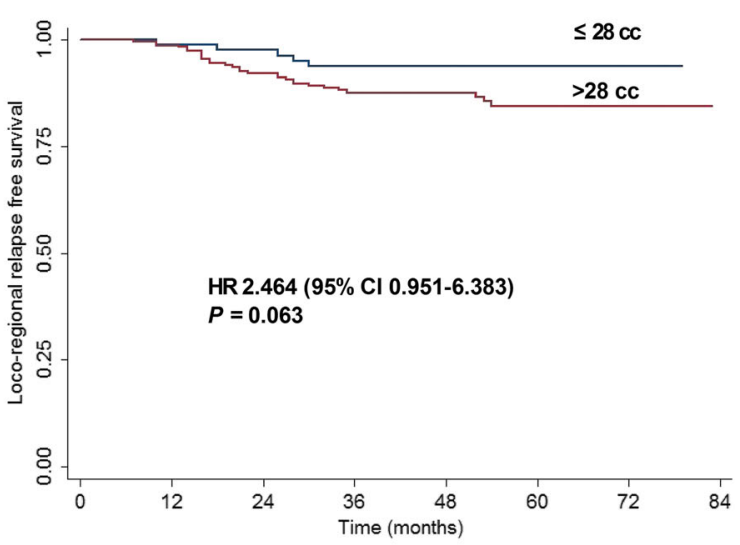

No. at risk:

\begin{tabular}{|c|c|c|c|c|c|c|}
\hline$\leq 28$ cc 81 & 80 & 78 & 73 & 63 & 42 & 17 \\
\hline & 217 & 189 & 162 & 98 & 49 & 19 \\
\hline
\end{tabular}

Fig. 4 Survival rates for the 316 patients with stage III-IVb stratified by TTV. a Failure-free survival. b Overall survival. c Distant metastasis-free survival. d Loco-regional relapse-free survival

rates decrease with increasing tumor volume in patients with NPC treated by 2D-RT or IMRT.

Multivariate analyses showed TTV was an independent prognostic factor for FFS, OS, DMFS and LRRFS. In comparison, $\mathrm{T}$ category was an independent prognostic factor for OS, but not for FFS, DMFS or LRRFS. Furthermore, the only independent prognostic factor for LRRFS was TTV. Similar results were also observed in head and neck carcinomas: TTV was an independent variable, but $\mathrm{T}$ and $\mathrm{N}$ category were not independent prognostic variables unless the multivariate analyses did not include TTV [29]. In both this and the previous study, TTV appeared to be a more useful prognostic factor than the AJCC staging system. A large tumor volume may indicate a high potential for micro-metastasis, tumor hypoxia that promotes resistance to RT and chemotherapy, and an increased number of cancer clone cells to be killed [30, 31].

\section{Prognostic significance of the TTV in loco-regionally advanced NPC}

This study also demonstrated that a large TTV was associated with poor FFS, OS and DMFS in stage III-IVb NPC. The TTV was also an independent prognostic factor for FFS, OS and DMFS in this group of patients. Our previous studies confirmed that MPTD is an independent prognostic variable in stage T3-T4 NPC $[25,26]$. These findings indicate that although loco-regionally advanced disease is usually associated with a high risk of 
Table 3 Multivariate analyses of prognostic factors in 316 patients with stage III-IVb

\begin{tabular}{lllll}
\hline Endpoint & Variable & HR & $95 \% \mathrm{Cl}$ & $P$-value \\
\hline FFS & TTV & 3.362 & $1.677-6.738$ & 0.001 \\
OS & Sex & 1.735 & $0.906-3.321$ & 0.096 \\
& Additional boost & 1.860 & $0.947-3.654$ & 0.072 \\
& Chemotherapy & 2.384 & $1.109-5.126$ & 0.026 \\
& N stage & 1.551 & $0.994-2.421$ & 0.053 \\
& TTV & 4.364 & $2.125-8.964$ & $<0.001$ \\
DMFS & TTV & 2.923 & $1.323-6.459$ & 0.008 \\
LRRFS & TTV & 2.464 & $0.951-6.383$ & 0.063 \\
\hline a & & &
\end{tabular}

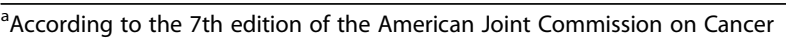
staging system; $H R$ hazard ratio, $C l$ confidence interval, FFS failure free survival OS overall survival, DMFS distant metastasis-free survival, LRRFS loco-regional relapse free survival, $T T V$ total tumor volume, $R T$ radiotherapy

treatment failure, patients with advanced stage disease and a small tumor size may have a better prognosis.

Previous studies indicated the PTV was a significant prognostic factor for local control in NPC [20]. Sze et al. reported the risk of local control was estimated to decrease by $1 \%$ for every $1 \mathrm{~cm}^{3}$ increase in the PTV [16]. In this study, for patients with stage III-IVb NPC, TTV was only just statistically significant in LRRFS analysis. The main reasons for this observation may be as follows: first, with the development of IMRT, LRRFS has increased compared to patients treated with 2D-RT and 3-dimensional conformal radiation therapy [7, 8] and secondly, only 81 patients in the stage III-IVb group had a TTV $\leq 28 \mathrm{~cm}^{3}$. Therefore, this trend needs to be confirmed by analysis of a larger sample.

\section{Prognostic validity of adding TTV to clinical stage}

The combination of TTV and clinical stage was superior to clinical stage alone for predicting treatment failure. Guo et al. reported prognostic assessment could be

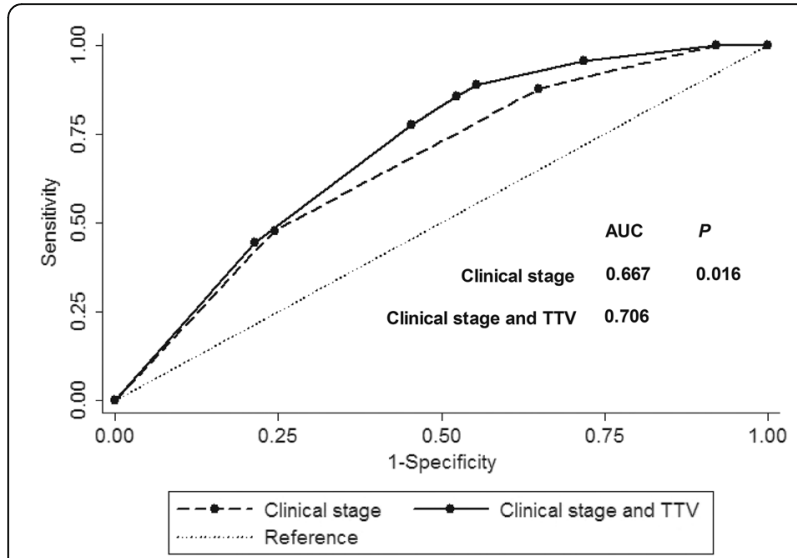

Fig. 5 ROC curves for all patients stratified by clinical stage combined with TTV or clinical stage improved by combining the PTV with the current T classification criteria [21]. Our previous study also showed inclusion of the MPTD improved the prognostic value of the current $\mathrm{T}$ classification criteria [25]. Therefore, the current staging system for patients with NPC could be refined by incorporating tumor size.

In the clinic, the treatment strategy for NPC is mainly based on the name staging system, which lacks indexes related to tumor burden. TTV closely reflects tumor burden and is easily obtained from the IMRT planning system. As a large TTV was associated with a high incidence of treatment failure, patients with a large TTV may benefit from more aggressive treatment. For instance, adding induction chemotherapy, including cisplatin, fluorouracil, and docetaxel (TPF), to concurrent chemoradiotherapy could significantly improve FFS in locoregionally advanced NPC [32].

\section{Conclusions}

This is the first evaluation of the prognostic value of the TTV in NPC, and reveals the TTV is an important prognostic factor for treatment outcomes in patients treated with IMRT. Incorporation of the TTV could help to refine the prognostic validity of the current staging system for NPC. Patients with a large TTV had a poor prognosis and may benefit from more aggressive treatment. However, this was a retrospective study of consecutive patients who received different chemotherapy regimens, which may have affected the treatment outcomes. Furthermore, this analysis was based on single-institution data, and needs to be confirmed via large-cohort multicenter studies.

\section{Abbreviations \\ AJCC: American Joint Commission on Cancer staging system; AUC: area under the curve, 2D, two-dimensional; CTV: clinical target volume; DMFS: distant metastasis-free survival; FFS: failure free survival; IMRT: intensity-modulated radiation therapy; LRRFS: loco-regional relapse free survival; MPTD: maximum primary tumor diameter; MRI: magnetic resonance imaging; NPC: nasopharyngeal carcinoma; NTV: nodal tumor volume; OS: overall survival; PTV: primary tumor volume; ROC: Receiver operating characteristic; RT: radiotherapy; TTV: total tumor volume}

\section{Acknowledgements}

The authors acknowledge the department of medical records for permission to access the linked databases.

\section{Funding}

This work was supported by grants from the National Natural Science Foundation of China (No. 1473233), the Science Foundation from the Sci-Tech Office of Guangdong Province, China (No. 2016A020215083) and the Science Foundation from the Sci-Tech Office of Foshan City, China (No. 2015AB00294).

Availability of data and materials

The datasets used and analysed during the current study were available from the corresponding author on reasonable request.

Authors' contributions

SBL and JJT contributed to the conception and design of the study, data acquisition, data analysis, data interpretation and the draft of the manuscript. $X F H, X L Y, M L$ and XNF carried out the acquisition and interpretation of data. 
DSL performed the data analysis and data interpretation. YC and LWF contributed with the conception and design of the study, data acquisition, data interpretation and critical edit of the manuscript. All authors read and approved the final manuscript.

\section{Ethics approval and consent to participate}

The study was approved by the Institutional Review Board of First People's Hospital of Foshan Affiliated to Sun Yat-sen University. It was a retrospective analysis of routine data and thus we were granted an exemption from requiring written informed consent.

\section{Consent for publication}

Not applicable.

\section{Competing interests}

The authors declare that they have no competing interests.

\section{Publisher's Note}

Springer Nature remains neutral with regard to jurisdictional claims in published maps and institutional affiliations.

\section{Author details \\ 'State Key Laboratory of Oncology in South China, Sun Yat-sen University Cancer Center, Collaborative Innovation Center for Cancer Medicine, Guangzhou 510060, China. ${ }^{2}$ Department of Radiation Oncology, Cancer Center, First People's Hospital of Foshan Affiliated to Sun Yat-sen University, Foshan, China. ${ }^{3}$ Department of Medical Statistics, First People's Hospital of Foshan Affiliated to Sun Yat-sen University, Foshan, China. ${ }^{4}$ Department of Radiation Oncology, The First Affiliated Hospital of Sun Yat-Sen University, Guangzhou, China}

\section{Received: 28 December 2016 Accepted: 11 July 2017}

\section{Published online: 28 July 2017}

\section{References}

1. Torre LA, Bray F, Siegel RL, Ferlay J, Lortet-Tieulent J, Jemal A. Global cancer statistics, 2012. CA Cancer J Clin. 2015;65:87-108

2. Zhang LF, Li YH, Xie SH, Ling W, Chen SH, Liu Q, et al. Incidence trend of nasopharyngeal carcinoma from 1987 to 2011 in Sihui County, Guangdong Province, South China: an age-period-cohort analysis. Chin J Cancer. 2015;34:350-7.

3. Blanchard P, Lee A, Marguet S, Leclercq J, Ng WT, Ma J, et al. Chemotherapy and radiotherapy in nasopharyngeal carcinoma: an update of the MAC-NPC meta-analysis. Lancet Oncol. 2015;16:645-55

4. Lee AW, Ma BB, Ng WT, Chan AT. Management of Nasopharyngeal Carcinoma: current practice and future perspective. J Clin Oncol. 2015:33:3356-64

5. Yu E, O'Sullivan B, Kim J, Siu L, Bartlett E. Magnetic resonance imaging of nasopharyngeal carcinoma. Expert Rev Anticancer Ther. 2010;10:365-75.

6. Lee AW, Ngan RK, Tung SY, Cheng A, Kwong DL, Lu TX, et al. Preliminary results of trial NPC-0501 evaluating the therapeutic gain by changing from concurrent-adjuvant to induction-concurrent chemoradiotherapy, changing from fluorouracil to capecitabine, and changing from conventional to accelerated radiotherapy fractionation in patients with locoregionally advanced nasopharyngeal carcinoma. Cancer. 2015:121:1328-38.

7. Lai SZ, Li WF, Chen L, Luo W, Chen YY, Liu LZ, et al. How does intensitymodulated radiotherapy versus conventional two-dimensional radiotherapy influence the treatment results in nasopharyngeal carcinoma patients? Int J Radiat Oncol Biol Phys. 2011;80:661-8.

8. Peng G, Wang T, Yang KY, Zhang S, Zhang T, Li Q, et al. A prospective, randomized study comparing outcomes and toxicities of intensity-modulated radiotherapy vs. conventional two-dimensional radiotherapy for the treatment of nasopharyngeal carcinoma. Radiother Oncol. 2012;104:286-93.

9. Lin S, Pan J, Han L, Guo Q, Hu C, Zong J, et al. Update report of nasopharyngeal carcinoma treated with reduced-volume intensitymodulated radiation therapy and hypothesis of the optimal margin. Radiother Oncol. 2014;110:385-9.

10. Sun X, Su S, Chen C, Han F, Zhao C, Xiao W, et al. Long-term outcomes of intensity-modulated radiotherapy for 868 patients with nasopharyngeal carcinoma: an analysis of survival and treatment toxicities. Radiother Oncol. 2014:110:398-403.

11. Ng WT, Lee MC, Chang AT, Chan OS, Chan LL, Cheung FY, et al. The impact of dosimetric inadequacy on treatment outcome of nasopharyngeal carcinoma with IMRT. Oral Oncol. 2014;50:506-12.

12. Yi J, Huang X, Gao L, Luo J, Zhang S, Wang K, et al. Intensity-modulated radiotherapy with simultaneous integrated boost for locoregionally advanced nasopharyngeal carcinoma. Radiat Oncol. 2014;9:56.

13. Zhang H, Seikaly H, Nguyen NT, Abele JT, Dziegielewski PT, Harris JR, et al. Validation of metabolic tumor volume as a prognostic factor for oral cavity squamous cell carcinoma treated with primary surgery. Oral Oncol. 2016;57:6-14

14. Cottereau AS, Lanic $H$, Mareschal $S$, Meignan M, Vera $P$, Tilly $H$, et al. Molecular profile and FDG-PET/CT Total metabolic tumor volume improve risk classification at diagnosis for patients with diffuse large B-cell lymphoma. Clin Cancer Res. 2016:22:3801-9.

15. Rodeberg DA, Stoner JA, Garcia-Henriquez N, Randall RL, Spunt SL, Arndt CA, et al. Tumor volume and patient weight as predictors of outcome in children with intermediate risk rhabdomyosarcoma: a report from the Children's oncology group. Cancer. 2011;117:2541-50.

16. Sze WM, Lee AW, Yau TK, Yeung RM, Lau KY, Leung SK, et al. Primary tumor volume of nasopharyngeal carcinoma: prognostic significance for local control. Int J Radiat Oncol Biol Phys. 2004;59:21-7.

17. Wu Z, Gu MF, Zeng RF, Su Y, Huang SM. Correlation between nasopharyngeal carcinoma tumor volume and the 2002 International Union against Cancer tumor classification system. Radiat Oncol. 2013;8:87.

18. Lee N, Harris J, Garden AS, Straube W, Glisson B, Xia P, et al. Intensitymodulated radiation therapy with or without chemotherapy for nasopharyngeal carcinoma: radiation therapy oncology group phase II trial 0225. J Clin Oncol. 2009:27:3684-90

19. Edge SB, Compton CC. The American joint committee on cancer: the 7th edition of the AJCC cancer staging manual and the future of TNM. Ann Surg Oncol. 2010;17:1471-4.

20. Chua DT, Sham JS, Kwong DL, Tai KS, Wu PM, Lo M, et al. Volumetric analysis of tumor extent in nasopharyngeal carcinoma and correlation with treatment outcome. Int J Radiat Oncol Biol Phys. 1997;39:711-9.

21. Guo R, Sun $Y, Y u X L$, Yin $W J$, Li WF, Chen $Y Y$, et al. Is primary tumor volume still a prognostic factor in intensity modulated radiation therapy for nasopharyngeal carcinoma? Radiother Oncol. 2012;104:294-9.

22. Van den Brekel MW, Stel HV, Castelijns JA, Nauta JJ, van der Waal I, Valk J, et al. Cervical lymph node metastasis: assessment of radiologic criteria. Radiology. 1990;177:379-84.

23. Mao YP, Liang SB, Liu LZ, Chen Y, Sun Y, Tang LL, et al. The N staging system in nasopharyngeal carcinoma with radiation therapy oncology group guidelines for lymph node levels based on magnetic resonance imaging. Clin Cancer Res. 2008;14:7497-503.

24. Feng M, Wang W, Fan Z, Fu B, Li J, Zhang S, et al. Tumor volume is an independent prognostic indicator of local control in nasopharyngeal carcinoma patients treated with intensity-modulated radiotherapy. Radiat Oncol. 2013:8:208.

25. Chen $Y$, Hu XF, Wang Y, Chen HY, Yang L, Liu LZ, et al. Is maximum primary tumor diameter still a prognostic factor in patients with nasopharyngeal carcinoma treated using intensity-modulated radiotherapy? BMC Cancer 2015;15:305.

26. Liang SB, Deng YM, Zhang N, Lu RL, Zhao H, Chen HY, et al. Prognostic significance of maximum primary tumor diameter in nasopharyngeal carcinoma. BMC Cancer. 2013:13:260.

27. Sarisahin M, Cila A, Ozyar E, Yildiz F, Turen S. Prognostic significance of tumor volume in nasopharyngeal carcinoma. Auris Nasus Larynx. 2011;38:250-4

28. Lee CC, Ho HC, Lee MS, Hsiao SH, Hwang JH, Hung SK, et al. Primary tumor volume of nasopharyngeal carcinoma: significance for survival. Auris Nasus Larynx. 2008:35:376-80.

29. Johnson CR, Khandelwal SR, Schmidt-Ullrich RK, Ravalese J 3rd, Wazer DE. The influence of quantitative tumor volume measurements on local control in advanced head and neck cancer using concomitant boost accelerated superfractionated irradiation. Int J Radiat Oncol Biol Phys. 1995;32:635-41.

30. Lartigau $E$, Le Ridant AM, Lambin P, Weeger $P$, Martin L, Sigal R, et al. Oxygenation of head and neck tumors. Cancer. 1993;71:2319-25. 
31. Zhao B, Oxnard GR, Moskowitz CS, Kris MG, Pao W, Guo P, et al. A pilot study of volume measurement as a method of tumor response evaluation to aid biomarker development. Clin Cancer Res. 2010;16:4647-53.

32. Sun Y, Li WF, Chen NY, Zhang N, Hu GQ, Xie FY, et al. Induction chemotherapy plus concurrent chemoradiotherapy versus concurrent chemoradiotherapy alone in locoregionally advanced nasopharyngeal carcinoma: a phase 3, multicentre, randomised controlled trial. Lancet Oncol. 2016;17:1509-20.

Submit your next manuscript to BioMed Central and we will help you at every step:

- We accept pre-submission inquiries

- Our selector tool helps you to find the most relevant journal

- We provide round the clock customer support

- Convenient online submission

- Thorough peer review

- Inclusion in PubMed and all major indexing services

- Maximum visibility for your research

Submit your manuscript at www.biomedcentral.com/submit 